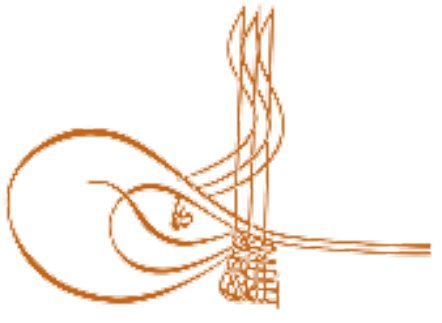

www.turkishstudies.net/language
Turkish Studies - Language and Literature

eISSN: $2667-5641$

Research Article / Araștırma Makalesi

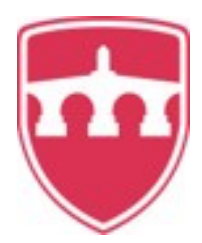

INTERNATIONAL

BALKAN

UNIVERSITY

Sponsored by IBU

\title{
“Les Mémoires D’un Âne”: Analyse Comparative Des Éléments Culturels De Ses Quatre Traductions En Turc*
}

\author{
"The Story of a Donkey": Comparative Analysis of the Cultural Elements of His Four Turkish \\ Translations
}

Filiz Tokalak $^{* *}$

\begin{abstract}
The field of translation of children's literature requires more special work than others. Since it is the child reader that is at stake, it is undeniable that this task requires much more attention and care. In this work, the four Turkish translations $(1940,1948,1959,2006)$ of one of the popular works, "The story of a Donkey", by the Countess of Ségur, a prosperous and popular writer of children's literature, have been selected among the other translations called (considered) adaptation on our part. While focusing on the importance of the translator's attitude during the translation process, the target-oriented approach (Riita Oittinen, Emer O'Sullivan, Toury) considered quite popular lately is criticized from the point of view of the literary, educational role of translation having children as the reader of the foreign culture. Using the classification of Göte Klingberg, E. Benveniste and the classification of Grit and Holmes, toponyms, anthroponyms and cultural elements (food, clothing, religion, money, hours, measurements) were analyzed according to the strategy of domestication and foreignization of Venuti. Several findings are made based on the language which differs from one translation to another reflecting the effects of their time according to Toury's theory. Always supporting the contribution of a work in a child's life (no matter what kind, an original work developing one's knowledge of one's own culture or a translation developing one's knowledge of a foreign culture), this source of richness, the foreign work, cannot be transmitted other than by adopting a faithful, source-oriented approach as much as possible in a translation of children's literature.
\end{abstract}

Structured Abstract: This is a descriptive work. It is part of the field of translation studies, particularly translation of children's literature. We have selected four different Turkish versions of the novel "The Story of a Donkey" by the Countess of Ségur, a distinguished author of the 19th century.

Certainly, as it should be the case in every work of children's literature, "The Story of a Donkey" is a rather rich book from the cultural point of view and it is also important to keep this cultural richness in the

\footnotetext{
${ }^{*}$ Bu çalışma yazarın doktora tezinden üretilmiştir.

** Arş. Gör. Dr., Hacettepe Üniversitesi, Edebiyat Fakültesi, Mütercim-Terümanlık Bölümü, Fransızca Mütercim Tercümanlık Anabilim Dalı.

Res. Assist. Dr., Hacetepe University, Faculty of Letters, Department of Translation and Interpreting, French Division ORCID 0000-0003-1613-2967

filiztokalak@hotmail.com
}

Cite as/ Atıf: Tokalak, F. (2020). "Les Mémoires D’un Âne ": analyse comparative des éléments culturels de ses quatre traductions en Turc, Turkish Studies - Language, 15(1), 443-462. https://dx.doi.org/10.29228/TurkishStudies.40423 Received/Geliş: 04 January/Ocak 2020

Accepted/Kabul: 24 March/Mart 2020 Checked by plagiarism software

Copyright (C) INTAC LTD, Turkey

Published/Yayın: 30 March/Mart 2020 CC BY-NC 4.0 
translation. We detect cultural notions, i.e. names of dishes, specific food peculiar to French culture, vocabulary specific to religion and religious rituals, daily life, customs not only of the villagers but also of the nobility, peculiar to France of the 1860s.

We were able to collect 24 editions completed in Turkish Republic period, 14 of which are translated by different translators, according to our research. On the other hand, we have not had the chance to evaluate the first two published in 1926, before the adoption of the new Turkish alphabet.

Our four translations into Turkish of the works entitled "Bir eşeğin hatıratı" (1940), "Bir eşeğin hatıraları" (1948), "Bir eşeğin anıları" (1959), "Bir eşeğin anıları" (2006), which have never been studies before, are analyzed comparatively and according to Venuti's strategies of domestication and foreignization. In this research, we focused preferably on elements including anthroponyms, place names and cultural elements. From the examples analyzed, we are able to depict the decisions, the attitude of translators in certain periods in Turkey. We would also like to point out that these are only a few representative examples that we have chosen to share. In addition, we intend to carry out another comparative work later on, examining the stylistic points reflecting the style of the writer.

From our analyses, when the time of translation is taken into account, we see that in the 1940s and 1950s, the Translation Bureau had set definite source-oriented standards, such as having to keep the spelling of the foreign language as it was. According to the analysis of the Turkish literary polysystem, the Bureau was placed at the center of the country's own literary polysystem. The private publishing houses were placed at the periphery of the Turkish literary polysystem. Indeed, they had decided to take into account standards such as "adapting the spelling of proper names to the target language".

If the translation of the toponyms of the four translations is to be evaluated, it should be pointed out that the first translator makes a spelling adaptation in the target language. This applies to the translation of anthroponyms as well. In fact, we can say that this is due to the literary polysystem of the country at that time. As for the last translator, he omitted all place names. Indeed, with this attitude, we consider that the translator does not intend to keep the strangeness at all, in other words, he does not want to capture the exoticism in his translation. Therefore, it is better to call him "target-oriented". As for the examples classified under the headings of food, religion, clothing, time and money, we generally notice a great lack of information about the cultural realities of the source culture for the target reader.

In the meantime, we have also interpreted the traces identified reflecting the effects of the time of the work's translation. The target-oriented attitude of the theorists is one of the reasons which motivated us to do this study in order to criticize their idea and to prove the missing, disadvantageous, incomplete literary and educational parts of the works of children's literature. Should we have a source-oriented or target-oriented approach? This question will be asked throughout our study.

Riita Oittinen, a target-oriented theorist, suggests that translators of children's literature should take full account of the target audience. In this case, she gives permission to make adaptations in the source text in order to remove the incomprehension (we believe that children are ready to understand everything as soon as it is explained to them) of the target audience. As for us, we consider that nothing should prevent us from behaving as target-oriented even in the translation of children's literature. It is obvious that the translator must address child readers correctly, however, we find that it is possible to do so by preserving foreign notions, while keeping the objective of teaching the novelty, the cultural richness of the work to the child reader, always based on the original state of the work. In fact, it should simply be remembered that a child is always inclined to learn new things, it is innate in him. So why should we worry?

By having a source-oriented attitude in the translation of children's literature, we may be forcing the translator to be more creative. Since this is one of the missions of children's literature, children learn new things, they have the opportunity to discover new worlds and develop knowledge. By selecting the correct words, the reader will have the same feeling and will learn the same language background as the reader of the original book. This is why we insist on the fact that a children's literature translator can react in a different way other than being target-oriented. If necessary, the translator can add small explanations, small pictures to facilitate the understanding of the new vocabulary. Consequently, the "child" reader will be able to enrich himself thanks to a translation. 
In conclusion, and in view of these comments, we hope to have made a contribution to the work on the translation of children's literature by illustrating our vision: the importance of being source-oriented in this area, which we will describe as delicate, while taking the child reader at the center of learning, either through a book called "original" or simply called "translation".

Keywords: Translation of Children's Literature, Target-Oriented, Source-Oriented, Domestication, Foreignization, Cultural Elements.

Résumé: Le domaine de la traduction de la littérature de jeunesse exige un travail particulier car c'est de l'enfant lecteur dont il est question. Raison pour laquelle, dans ce travail, les quatre traductions en turc (1940, 1948, 1959, 2006) de l'une des œuvres populaires, "Les mémoires d'un Âne", de la Comtesse de Ségur, écrivain prospère et populaire de la littérature de jeunesse ont été sélectionnées parmi les autres traductions considérées plutôt comme des adaptations de notre part. Tout en se focalisant sur l'importance de l'attitude du traducteur pendant le processus de traduction, l'approche cibliste, (Riita Oittinen, Emer O'Sullivan, Toury) considérant qu'elle est assez populaire ces derniers temps, est critiquée du point de vue du rôle littéraire et éducatif que la traduction peut avoir auprès de l'enfant lecteur. En se servant de la classification de Göte Klingberg, E. Benveniste et la classification de Grit et Holmes, les toponymes, les anthroponymes et les éléments culturels (la nourriture, les vêtements, la religion, l'argent, les heures, les mesures) ont été analysés d'après la stratégie de domestication et de foreignisation de Venuti. Plusieurs constats ksont établis à partir de la langue qui diffère d'une traduction à l'autre reflétant les effets de leur époque d'après la théorie de Toury. Soutenant toujours la contribution d'une œuvre dans la vie d'un enfant (peu importe que ce soit une œuvre originale, développant ses connaissances de sa propre culture ou une traduction développant ses connaissances d'une culture étrangère), cette source de richesse, l'œuvre étrangère, ne peut se transmettre autrement qu'en une approche fidèle, sourcière dans la mesure du possible dans une traduction de littérature jeunesse.

Mots Clés : Traduction de Littérature Jeunesse, Cibliste, Sourcier, Domestication, Foreignisation, Éléments Culturels.

\section{Introduction}

"Les mémoires d'un Âne", l'œuvre dont nous allons comparer les traductions en turc, fait partie des œuvres de la littérature de la jeunesse. Dans un premier temps, nous aborderons la notion de la littérature de jeunesse et nous partagerons les pensées principales des théoriciens très populaires du domaine.

Ewers définit brièvement les exemples de production de la littérature de jeunesse (2009): 1. Étant donné que ce sont des productions visant les adultes, les jeunes lisent aussi ces œuvres originales, sans manipulation demandée ni par la maison d'édition ni par l'auteur ("recommended reading for children and young people"). 2. Ce sont des productions réalisées visant les adultes mais conseillées aussi aux jeunes. Elles sont attrayantes et grâce à cela, de nouvelles éditions visent le nouveau lecteur sous forme de diffusion spéciale ("children's only publishing"). 3 Il existe des textes écrits pour les adultes. Ce sont des textes qui subissent maintes modifications afin de répondre aux besoins de leur nouveau public, voire les jeunes lecteurs. Nous pouvons évoquer les classiques qui sont adaptés à la jeunesse comme exemple ("children's editing"). 4. On appelle le quatrième groupe "original children's writing" en anglais. On y vise à transférer implicitement l'objectif littéraire aux enfants et aux jeunes lecteurs. L'écrivain a pour priorité de rédiger pour transférer des messages à son propre public.

Comme Erten (2011) l'affirme dans son livre intitulé "Çocuk yazını çevirisine yaklaşımlar", on remarque une aptitude générale parmi les théoriciens "Riita Oittinen, Tiina Puurtinen et Emer O'Sullivan" et aussi parmi les traducteurs de nos jours à être plutôt "ciblistes". Nous pouvons aussi ajouter Toury dans ce groupe. 
Riitta Oittinen est classée parmi les théoriciens les plus connus sur le terrain de la traduction de la littérature de jeunesse. Elle fait partie des soutenants de l'approche fonctionnaliste. Elle trouve que la traduction de la littérature de jeunesse est un acte culturel visant à combler une lacune, un besoin spécifié à l'avance (Pederzoli, 2012). Partageons ce que pense Oittinen à propos du domaine de la traduction de la littérature de jeunesse: "Les deux notions, la situation et l'objectif, sont les deux points propres à chaque traduction en elle-même. Les traducteurs ne traduisent jamais les mots en dehors de leur contexte. Ils doivent considérer les mots dans leur contexte" (Oittinen, 2000: 3$)^{1}$. En effet, elle souligne la notion de "contexte" dans le processus de traduction. Choisir le bon mot est un à priori, par contre en gardant toujours le souci de pouvoir préserver le même sens dépendant du contexte.

D'après Oittinen, la traduction jeunesse doit correspondre aux règles des fonctionnalistes. Par exemple, on doit se concentrer à traduire en considérant le besoin et la volonté du public visé. Ainsi, tant que ce que vous faites sert le public visé, on n'hésite pas à faire des manipulations dans le texte original. Oittinen accepte le fait que tout traducteur possède un bagage linguistique, culturel et enfantin spécifique. Ainsi, elle n'est pas contre la contribution réalisée par le traducteur dans l'œuvre de départ pendant le processus de traduction. Elle défend aussi l'idée selon laquelle le traducteur doit s'intégrer dans l'univers de l'enfant et doit y faire "le carnaval". Le traducteur doit prendre en considération les paroles des enfants de son entourage (Oittinen citée par Erten, 2011: 65).

Nous sommes du même avis qu'Oittinen. Il est indéniable que le traducteur est obligé de bien connaître son public visé. Nous ne pouvons imaginer le contraire. Par contre, nous tenons toujours compte du fait que le traducteur doit pouvoir s'adresser à son lecteur en ayant l'idée de garder l'étrangeté sous l'objectif de l'apprentissage de nouvelles choses à l'enfant et en respectant le plus possible l'originalité de l'œuvre de départ. Quand nous pensons à l'objectif éducatif d'une œuvre de littérature jeunesse, nous préférons bien évidemment nous conformer fidèlement à l'œuvre originale.

Gideon Toury et Itamar Even-Zohar font des études sur la théorie du polysystème; ces deux théoriciens se concentrent à priori sur le système littéraire et les normes des pays du texte de départ et du texte d'arrivée (Rakova, 2014). Gideon Toury affirme que le traducteur doit respecter les normes du pays de la culture cible. D'après la norme initiale, le traducteur doit faire un choix: il doit être soit sourcier soit cibliste. Cette norme désigne l'attitude du traducteur. Ces deux théoriciens s'interrogent aussi sur les dimensions culturelles, sociales, économiques et politiques pouvant influencer le traducteur. Oséki- Dépré souligne le fait que "les domaines littéraires et sociaux s'interpénètrent à travers les institutions littéraires, les idéologies, les maisons d'édition, la critique, les groupes littéraires ou toutes autres formes capables d'imposer les goûts et les normes" (Oséki- Dépré,1999: 62-63).

Après avoir évoqué les différentes approches des théoriciens concernant la littérature de jeunesse, nous souhaiterions apporter quelques précisions sur la contribution que notre travail va essayer d'apporter au domaine de la littérature de jeunesse. Il s'agit d'un travail desrciptif. Il fait partie du domaine de la traductologie, particulièrement de la traduction de la littérature jeunesse. Nous avons sélectionné quatre versions différentes en turc du roman intitulé "Les mémoires d'un âne" de la Comtese de Ségur, une femme de lettres renommée du XIX ${ }^{\mathrm{e}}$ siècle.

Nous avons pu rassembler 24 éditions en Turquie dont 14 sont traduites par différents traducteurs. Par contre, nous n'avons pas eu l'occasion d'évaluer les deux premières publiées en 1926, avant l'adoption du nouvel alphabet turc.

Nos quatre traductions en turc, "Bir eşeğin hatıratı" (1940), "Bir eşeğin hatıraları" (1948), "Bir eşeğin anıları" (1959), "Bir eşeğin anıları" (2006), réalisées à différentes époques n'ont jamais été, à notre connaissance, étudiées jusqu'à ce jour. Ces traductions sont analysées comparativement et ce, d'après les stratégies de domestication et d'étrangéisation (foreignisation) de Venuti. Entre temps, nous avons aussi interprété les indices reflétant les éléments de l'époque à laquelle la traduction a été effectuée. L'attitude

\footnotetext{
${ }^{1}$ Citation traduite par l'auteur de l'article.
} 
cibliste des théoriciens nous a-motivés à réaliser ce travail afin de critiquer leurs idées et de mettre en exergue les parties manquantes, inadéquates du point de vue littéraire et éducatif des œuvres de la littérature de jeunesse. La question: faut-il être sourcier ou cibliste? se posera tout au long de ce travail.

Dans cette recherche, nous nous sommes focalisés de préférence sur les éléments comprenant les anthroponymes, les toponymes et les éléments culturels. À partir des exemples qui seront analysés, nous allons essayer de dépeindre les décisions et les positions que les traducteurs ont adoptées lors de ces traductions réalisées à différentes époques. Nous voudrions préciser aussi que ce ne sont que quelques exemples représentatifs que nous avons choisis de partager.

Dans la littérature de jeunesse, les stratégies adoptées par les traducteurs sont, généralement, les stratégies de domestication et d'étrangéisation. Il nous semble intéressant de les évoquer avant de passer à la partie de l'analyse.

\section{Stratégie de la Domestication/l'Étrangéisation (la Foreignisation)}

Les termes de visibilité et d'invisibilité sont rencontrés pour la première fois dans l'œuvre de Lawrence Venuti, "The Translator's Invisibility: A History of Translation" (1995). Du mot "invisible", nous entendons que la trace du traducteur apparait comme invisible dans son acte de traduire. Quant à Gideon Toury et Itamar Even-Zohar, ils travaillent sur la notion de visibilité du traducteur à l'aide de leurs analyses descriptives (Doğru, 2011).

En effet, le traducteur présente "l'étranger" à toute personne de tout niveau dans la société. Étant donné que ce dont il est question est une relation entre deux cultures tout à fait différentes, le traducteur a trois choix: il peut conserver l'original ou bien importer des mots étrangers et peut-être même pratiquer le néologisme. En d'autres termes, l'attitude du traducteur peut renforcer l'assimilation de l'étrangeté (les normes existantes) ou bien il y aura des innovations dans la langue et la culture" (Torres, 2012).

Les notions "le cultural turn", "le tournant cuturel" abordées dans la science de la traduction nous conduisent chez Venuti. On peut dire aussi que les analyses de ce dernier sont plus globales du point de vue traductologique (Gile, 2005: 248-250).

Venuti soutient l'idée que les textes appartenant à une culture appelée "faible" et traduits vers une culture appelée "forte" ont tendance à être traduits selon la stratégie de domestication. Autrement dit, traduire d'une façon naturelle pour le lecteur de la culture d'arrivée. Autrement dit, ceux-ci sont traduits en visant le lecteur de la culture d'arrivée. Quant aux textes appartenant à une culture forte et traduits vers une culture moins forte, ils ont plus de chance d'être transférés à l'aide de la stratégie de d'étrangéisation. Dans une telle situation, le traducteur garde les caractéristiques de la langue et de la culture du texte de départ.

Il nous parait notable de souligner que nous ne pouvons pas considérer les deux théoriciens (Venuti et Toury) de la même manière du fait que Venuti se sépare des traductologues de DTS (Desrciptive Translation Studies) alors que Toury analyse la traduction d'après la situation du texte en ajoutant un point de vue idéologique.

La stratégie de domestication met en avant la visibilité du traducteur. Ce qui donne au lecteur cible l'impression de lire une production de sa propre culture; effectivement, les éléments étrangers existant dans le texte original sont omis. Nous rencontrons souvent une adapatation de noms propres, de lieux, d'aliments ou d'expressions idiomatiques, etc., si parfaitement que le lecteur du texte d'arrivée pourrait ne pas se rendre compte qu'il est devant une traduction.

Le traducteur s'efforce à conserver le sens du texte et le registre de langue correspondant aux règles linguistiques de la langue et de la culture d'arrivée. Finalement, nous obtenons une œuvre, "un texte qui ne sent pas la traduction" (Alipui, 2004: 18). 
À ce propos, Venuti précise: "la fluidité peut être considérée en tant que stratégie discursive adaptée à la traduction domestique (...) en produisant l'effet de transparence, l'illusion que ce n'est pas une traduction" (Venuti, 1995: 61). Comme elle représente une stratégie sur la richesse authentique de la culture d'arrivée, cet acte est très important. Dans ce cas, le traducteur est invisible, il ne fait pas sentir que l'œuvre de production est une interprétation du texte de départ produit par le traducteur (Alipui, 2004).

Hélouise Debombourg considère que le traducteur adoptant la stratégie de domestication, ayant l'impression de faire "une bizarrerie de l'étranger" toujours sous l'objectif "de rendre le texte plus accessible" (Debombourg, 2011: para.6), supprime les mots perturbateurs qui provoquent une incompréhension de l'œuvre.

Dans la stratégie de foreignisation (étrangéisation), le traducteur ne vise pas à éliminer les notions culturelles puisque son but est de faire connaitre un nouveau monde au lecteur du texte d'arrivée. Les noms propres (noms de personnes et de lieux), les évènements culturels et historiques sont logiquement étrangers, inconnus et aussi intéressants.

Comme le précise Alipui (2004: 16) avec qui nous sommes tout à fait d'accord, en abordant la stratégie de foreignisation, le traducteur accomplit une deuxième mission. En effet, grâce à cette méthode, il réussit à placer l'auteur du texte original "au dessus" du traducteur avec pour l'objectif de présenter "les valeurs" et "la richesse" de la culture du texte de départ. De plus, "le traducteur réussit à être fidèle à l'auteur du livre original et nous considérons qu'il apporte aussi une contribution à la survie de l'œuvre telle qu'elle est" (Tokalak-Baltac1, 2017: 117).

Partageons les pensées de Cachin: "Si une certaine adaptation à la culture d'accueil est inéluctable, il ne faut pas qu'elle conduise à la neutralisation de ce qui est différent, à la négation de l'œuvre en tant qu'étrangère" (Citée par Debombourg, 2011: para.10). En interprétant les paroles de Cachin, nous voyons qu'il est possible de faire de petites manipulations faute de trouver d'autres solutions. Par contre, il souligne qu'il faut savoir limiter dans l'inquiétude de ne pas tuer l'originalité de l'œuvre.

Afin d'éviter la perte de l'originalité, nous pouvons trouver des solutions qui pourront nous aider telles qu'ajouter des explications succintes, des notes ou bien des images à la fin du livre.

La traductrice B. Stolt (Musset, citée par Debombourg, 2011: para.10) défend "l'authenticité" du texte. Elle considère que les enfants sont toujours prêts à accepter l'étrangeté car ils ont une curiosité qui les motive à connaître les nouveautés.

En effet, nous considérons aussi que l'enfant est toujours curieux d'informations et de nouveautés. Pendant sa lecture, rencontrer des réalités inconnues va attirer son attention et il va être poussé à la curiosité. Plusieurs questions peuvent apparaitre dans son esprit, l'incitant à chercher des réponses autour de lui.

Debombourg est partisane de la stratégie de foreignisation. Elle défend l'idée de faire comprendre à notre lecteur enfant, qu'il lit une traduction et non un livre de sa propre culture. Ainsi, l'enfant serait plus conscient de l'existence d'une autre langue et d'une autre culture que la sienne. Ce qui est à retenir, c'est que l'enfant s'ouvre au monde entier grâce à cela.

Admettons qu'avec la méthode de foreignisation, le traducteur soit visible en totalité. Il est chargé de faire découvrir un autre monde à son lecteur enfant dans sa propre langue. En abordant la technique de foreignisation, le traducteur devient très fidèle à l'auteur du texte de départ. Il ne se soucie pas de la compréhension par le public du texte d'arrivée.

\section{Écrivain et son CEuvre "Les mémoires d'un Âne"}

À l'époque du Second Empire et juste au début de la Troisième République, l'enfant a finalement eu accès à la littérature d'enfance et de jeunesse. Dans ces œuvres, on se concentre totalement sur 'l'enfant 
héros" dans les histoires. On se donne alors pour but d'enseigner la morale à l'aide des notions contraires comme "le bon ou le mauvais, le bonheur ou le malheur, la vérité ou le mensonge, etc". Nous pouvons aussi ajouter que c'est un moyen pour les adultes de connaitre l'état psychologique de leur enfant d'une manière plus détaillée (Escarpit, 2008: 243).

Nous pouvons considérer que notre œuvre d'analyse "Les mémoires d'un Âne" se compte dans ce groupe. Dans le livre, chaque histoire se termine avec des leçons positives mais par contre l'âne subit plusieurs revers.

Boutevin et Principalli affirment que c'est grâce à notre écrivain que les livres ont commencé à s'adresser réellement aux enfants (Boutevin et Principalli, 2008: 164). Sophie de Ségur se lance pour la première fois à 58 ans dans le monde de la littérature de jeunesse avec sa publication "Les nouveaux contes de fées" (1857). Avec son succès, Louis Hachette crée la collection connue "Bibliothèque rose" dans laquelle on trouve des histoires en illustrations, des albums et des bandes dessinées au milieu du $\mathrm{XIX}^{\mathrm{e}}$ siècle.

Avec son œuvre "Petites Filles Modèles" (1857), la Comtesse de Ségur a commencé à placer "l'enfant" comme personnage central dans un roman de littérature de jeunesse. Les autres auteurs qui sont attirés par l'enfance dans son intégralité vont suivre le chemin de la Comtesse de Ségur qui dépeint dans ses œuvres "la psychologie et la vie des enfants" sous de multiples points de vue (Escarpit, 2008: 239).

De même, le livre "Les mémoires d'un Âne" publié en 1860 suscite une curiosité avec son personnage inhabituel, notre "âne". Nous considérons que le personnage du livre, "l'âne" doit attirer l'attention non seulement des écrivains français, mais aussi de ceux des pays où l'on trouve ses traductions.

\section{Différentes Classifications des Éléments Culturels}

Nous pouvons mentionner Göte Klingberg qui est connu dans la traduction jeunesse avec une liste de référents culturels reformés d'après ses priorités sous "une approche prescriptive" (Pederzoli, 2012: 99). Il se concentre généralement sur une théorie démarrant des adaptations d'après un aspect culturel des productions de littérature jeunesse. Il est vrai que nous faisons souvent face à des problèmes traductologiques dans ces aspects culturels nommés aussi "cultural context adaptations" par les anglophones. Dans cette analyse, nous nous sommes servis de la classification de Göte Klingberg:

1. Les références littéraires 2 . Les langues étrangères dans le texte source 3 . Références à la mythologie et à des croyances populaires 4 . Le bagage historique, religieux et politique 5 . Construction et ameublement, la nourriture 6. Les traditions, les mœurs ..., les jeux, activités ludiques 7. Flore et Faune 8. Prénoms, titres, le nom des animaux domestiques, le nom des objets 9. Les noms géographiques 10. Poids et mesures (Klingberg, 1986, citée par Pederzoli, 2012: 99). ${ }^{2}$

Comme le précise Pederzoli (2012), il faut absolument souligner que Klingberg ne désire pas aborder toutes ces stratégies en une seule fois. Son but est de démontrer les cas possibles. Par contre, il est évident qu'il ne veut pas utiliser fréquemment la stratégie d'adaptation. En effet, il défend la préservation de l'originalité de l'œuvre (l'étrangéisation), et logiquement, il se pose contre l'idée de faire disparaitre l'étrangeté (la domestication).

À vrai dire, il existe maintes classifications. En complément des notions déterminées par Göte Klingberg, nous avons aussi un autre critère comme le tableau préparé par E. Benveniste et la classification de Grit et Holmes:

\footnotetext{
${ }^{2}$ Citation traduite par l'auteur de l'article.
} 


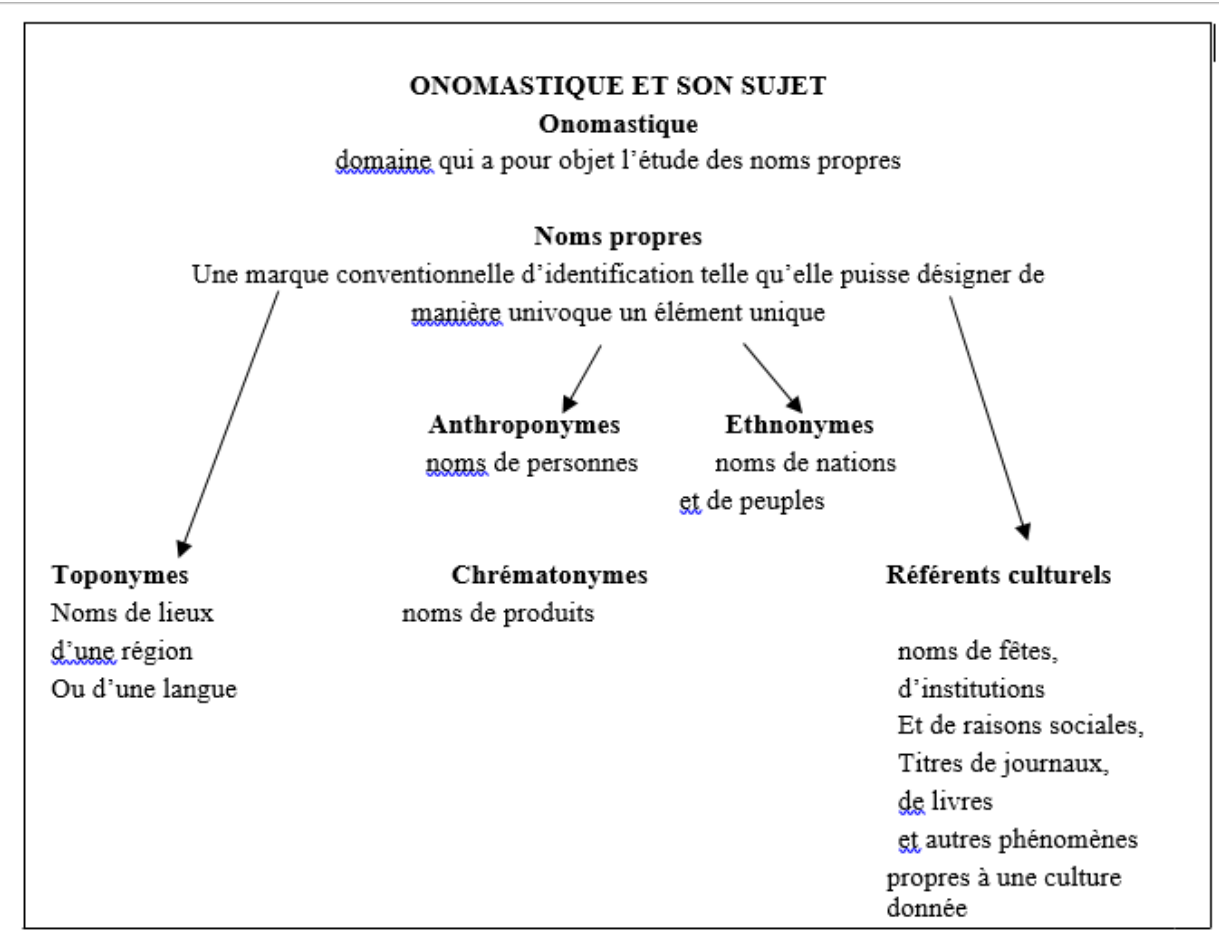

Shéma 1: L'Onomastique et son sujet. "Problèmes de linguistique générale 2", Benveniste, E. (1974: 200).

Durant l'analyse des quatre traductions de l'œuvre "Les mémoires d'un Âne", nous nous sommes concentrés plutôt sur trois grands axes: les anthroponymes, les toponymes et les éléments culturels. Nous allons maintenant partager quelques exemples considérés comme pertinents, représentant les stratégies des traducteurs.

\section{Analyse De Quelques Exemples Comparés Des Quatre Traductions De "Les Mémoires D'un Âne" En Turc}

\section{Traduction des Anthroponymes}

Pour réaliser ce travail, nous nous sommes inspirés des stratégies de plusieurs théoriciens. En ce qui concerne les stratégies dans la traduction des noms propres, il existe des noms qui restent tels quels dans la langue source et dans la langue cible. Nous pensons que cette réalité ne cause pas de problème.

Concernant la traduction des anthroponymes, Witteveen (2012: 33) cite trois stratégies:

“ - Maintenir le nom tel quel (capt'n Cook $\rightarrow$ le capitaine Cook), quand le nom propre renvoie à une entité (personne réelle) universellement connue sous cette dénomination. - Adapter orthographiquement le nom de la personne à la langue cible (Laure $\rightarrow$ Laura). - Traduire le nom dans la langue de la culture cible (Martin $\rightarrow$ Maarten)".

Dans le cas de noms propres ayant leurs équivalents dans les langues étrangères, le traducteur des textes littéraires peut néanmoins maintenir l'anthroponyme d'origine pour préserver la couleur locale du texte source.

Jan G. Witteveen souligne qu'en aucun cas nous ne pouvons traduire les noms propres. Par contre, s'il n'est pas possible d'écrire l'équivalent du nom propre dans la culture cible, il suggère de recourir à une adaptation. De même, il déclare que "pour préserver l'impression du lecteur qui lit un texte qui se déroule dans une autre culture, il est souhaitable de ne pas traduire les noms propres de 
personnages" (Witteveen, 2012: 34). Nous partageons aussi l'idée selon laquelle il faut absolument laisser les indices reflétant l'étrangéité et l'exotisme pour l'enfant.

Si préserver la couleur locale de la narration fait partie de nos objectifs, nous estimons qu'il est préférable d'employer la stratégie de non-traduction pour les noms de personnes. Il est toutefois bien entendu que si nous ne voulons pas compliquer les choses ni forcer les moyens, nous pouvons faire appel à l'adaptation orthographique ou phonétique avec l'équivalent exact dans la langue d'arrivée (Witteveen, 2012).

Tableau 1: Exemple 1 au Nom Propre

\begin{tabular}{|c|c|}
\hline \multirow[b]{2}{*}{ Livres } & \multirow{2}{*}{ Extraits } \\
\hline & \\
\hline T.S. & $\begin{array}{l}\text { (...) « Pauvre bête! Dit -elle enfin, tu n'as pas l'air méchant. Si tu } \\
\text { n'appartiens à personne, je serais bien contente de t'avoir pour remplacer } \\
\text { mon pauvre vieux Grison, mort de vieillesse. Je pourrais continuer à gagner } \\
\text { ma vie en vendant mes légumes au marché (...) (p.23) }\end{array}$ \\
\hline TT1 & $\begin{array}{l}\text { Biraz sonra, - zavallı hayvan! - dedi, - halinden iyi huylu olduğu } \\
\text { Anlaşılıyor. Eğer sahibin yoksa ben seni geçen gün ölen (Grizon) umun } \\
\text { yerine koyayım. Bu suretle yine eskisi gibi sebze satarak seninle geçinir, } \\
\text { giderim(p.21). }\end{array}$ \\
\hline TT2 & $\begin{array}{l}\text { Nihayet, "zavallı hayvan! Senin fena bir halin yok," dedi. Eğer sen } \\
\text { Kimsenin değilsen, ihtiyarlıktan ölen, benim zavallı ihtiyar (Grizon) umun } \\
\text { yerine sana sahip olmakla çok sevineceğim. Sebzelerimi pazara götürüp } \\
\text { satarak hayatımı kazanmağa devam edeceğim. (...) (p.17) }\end{array}$ \\
\hline TT3 & $\begin{array}{l}\text { (...) Zavallı hayvan, dedi, kötü bir mahlûka benzemiyorsun. Sahibin yoksa, } \\
\text { ihtiyarlıktan ölen zavallı Grizon'umun yerine seni yanıma almakla } \\
\text { memnun olacağım. Sebzelerimi pazarda satarak hayatımı kazanmağa } \\
\text { devam edebileceğim. (...) (p.13) }\end{array}$ \\
\hline TT4 & $\begin{array}{l}\text { Kadın sonunda: - Zavallı hayvancık! Sen de hiç kötü bir yaratığa } \\
\text { benzemiyorsun. Sahibin yoksa, ihtiyarlıktan ölen Kırçılımın yerine seni } \\
\text { alıkoyarım. Yine pazarda sebzelerimi satarak, hayatımı kazanmağa devam } \\
\text { ederim! (...) (p.9) }\end{array}$ \\
\hline
\end{tabular}

L'auteur écrit le mot "Grison" qui signifie dans la langue d'autrefois "un âne" (Le Nouveau Petit Robert, 1968: 1051). Les trois premiers traducteurs ont écrit le mot "Grison" dans l'orthographe de l'alphabet turc. Autrement dit, les traducteurs ont réalisé une adaptation orthographique dans le nom de l'animal dans la langue d'arrivée. Ils utilisent la stratégie de "foreignisation".

Par conséquent, nous comprenons que les trois traducteurs sont fidèles au texte de départ. Ils optent pour l'approche de "foreignisation". Quant au quatrième traducteur, il choisit le mot "kırçıl"qui signifie "kırçıllaşmaya başlamış, kır renkli" (Eren, 1988: 853) dans la langue d'arrivée. Ce mot a pour sens "gris, mêlé de blanc; adj de couleur grise (chien etc.)" (Kocabay, 2001: 631). Il préfère traduire le mot dans la langue d'arrivée. En d'autres termes, le mot porte le même sens dans la langue d'arrivée. Mais, dans ce cas, le traducteur fait disparaître les éléments essentiels qui créent l'étrangéité de l'œuvre. Ce faisant, il ne préserve ni l'exotisme ni la richesse de l'œuvre.

Tableau 2: Exemple 2 au Nom Propre

\begin{tabular}{ll}
\hline Livres & \multicolumn{1}{c}{ Extraits } \\
\hline T.S. & Camille: - J'ai eu peur d'une grenouille qui m’a sauté sur le pied. (p.93) \\
TT1 & Kamil: - Ayağıma bir kurbağa atladı da ondan korktum. (p.138) \\
TT2 & Camille: - Ayağımın üzerine sıçrayan kurbağa beni korkuttu. (p.140) \\
TT3 & Camille: - Ayağımın üzerine atlayan bir kurbağadan korkuyorum. (90) \\
TT4 & Camille: - Ayağımın üzerinden bir kurbağa atladı! (114) \\
\hline
\end{tabular}

Le premier traducteur fait une adaptation dans la traduction du mot "Camille". Il écrit "Kamil". Avec une adaptation orthographique, il doit échanger la dernière lettre "l" par un "y" pour pouvoir prononcer le mot correctement. Le mot "Kamil" est un nom masculin. Or, ce prénom est utilisé pour une fille dans cette œuvre (Sachons que le nom Camille est aussi utilisé pour un garçon dans la langue française). Si le lecteur cible comprend mal, s'il prend "Kamil" pour un garçon, cela 
peut causer un malentendu chez l'enfant. Comme ce dernier fait des efforts pour comprendre le sens de l'histoire, cela peut créer des confusions. En d'autres termes, le traducteur doit être très attentif et considérer que tout détail peut causer de mauvais effets du point de vue traductologique chez l'enfant lecteur.

Nous devons aussi noter qu'à l'exception du mot "Kamil", le premier traducteur a bien transmis tous les noms propres du livre dans la langue cible.

Précisons que dans les années 1940-1950, le Bureau de traduction avait imposé des normes définies. Ce bureau était sourcier: par exemple, il demandait de garder l'orthographe de la langue étrangère telle quelle. D'après l'analyse du polysystème littéraire turc, ce bureau était placé au centre du polysystème littéraire du pays même. On est alors amené à se poser la question: dans ce contexte, peut-on savoir comment se situent les maisons d'éditions privées et quels types de stratégies emploient-elles?

À cette époque, les maisons d'éditions privées étaient placées à la périphérie dans le polysystème littéraire turc. C'est la raison pour laquelle elles avaient décidé de tenir compte des normes comme "l'adaptation de l'orthographe des noms propres à la langue d'arrivée".

Si nous considérons nos quatre traductions imprimées par des maisons d'édition privées à la même époque, nous pouvons aisément argumenter les raisons des stratégies qu'elles ont employées.

\section{Traduction des Toponymes}

Paliczka affirme que d'habitude "on ne traduit pas les noms de places, rues, quartiers, parcs, restaurants, cafés et autres lieux publics. Cependant il existe un bon nombre d'exemples dont l'usage a créé des équivalents en langues étrangères" (Paliczka, citée par Witteveen, 2012: 36). Donc, il n'est pas commun de traduire "les Champs Élysées et le cimetière du Père Lachaise" (Witteveen, 2012: 36). Généralement, ce que les traducteurs préfèrent c'est garder les noms géographiques tels qu'ils sont. Par contre, s'il existe un équivalent préférable, ils peuvent manipuler. On peut aussi prendre en compte les adaptations orthographiques (Witteveen, 2012).

S'il faut évaluer la traduction des toponymes des quatre traductions, il faut préciser que le premier traducteur fait une adaptation orthographique dans la langue cible. Cela est valable pour la traduction des anthroponymes aussi. Nous pouvons affirmer que ceci est dû au système du polysystème littéraire du pays à cette époque-là. Quant au dernier traducteur, il a omis tous les noms de lieu. En effet, avec cette attitude, nous considérons que le traducteur n'a pas du tout l'intention de garder l'étrangéité. Autrement dit, il ne veut pas faire apparaître l'exotisme dans sa traduction. Il nous apparaît définitivement comme "cibliste".

Tableau 3: Exemple 1 au Nom de Lieu

\begin{tabular}{ll}
\hline Livres & \multicolumn{1}{c}{ Extraits } \\
\hline T.S. & - Des gens de Laigle. On raconte que l'âne de la ferme des Haies a été emporté et \\
TT1 & dévoré dans la forêt (p.19). \\
& - L Legl » kasabalılar. « He » lerin çiftliğindeki eşek te birkaç gündür ortadan \\
TT2 & kaybolmuş; paralandığına şüphe yok (p.18). \\
& -Laigle şehrinde oturanlar, Haies çiftliğine ait eşeğin ormanda parçalandığını \\
TT3 & söyliyorlar (p.14). \\
& -Laigle'de oturanlar. Haies çiftliğinin eşeğinin kurtlar tarafindan götürülüp \\
TT4 & ormanda parçalandığı söyleniyor (p.11). \\
& -SSehirden tanıdıklar. Çitler Çiftliği’nin eşeğini alıp götürmüşler de ormanda \\
& parçalamışlar (p.7). \\
\hline
\end{tabular}

Dans la première traduction, nous constatons une adaptation orthographique du nom de la ville "Laigle" transféré dans la langue d'arrivée comme "Legl". On voit toujours l'idée du traducteur qui veut sauvegarder l'étrangéité.

Par ailleurs, dans la première traduction, nous entendons que le mot "Haies" signifie un groupe de personnes. Par contre, dans les autres traductions, nous n'apercevons pas cette petite nuance informative particulière due à la langue étrangère. D'autre part, de même qu'on parle de Laigle, la ville de l'auteur, la 
ferme des Haies devait être un quartier de la ville. À l'époque de la Comtesse, la ville ne devait pas être très développée et de ce fait, "Les Haies" devait être un lieu-dit ou un hameau, petit coin écarté du village.

Le troisième traducteur garde l'étrangéité. Il se sert de la stratégie de la foreignisation. Nous le mettons directement dans le groupe "sourcier".

Le quatrième traducteur supprime le nom de la ville "Laigle". On dirait qu'il ne veut même pas le prononcer. Alors que, pour le terme "La ferme des Haies", il choisit de rédiger l'équivalence dans la langue du public cible en faisant disparaître l'expression française. Autrement dit, il emploie la stratégie de la domestication.

Nous ne remarquons aucune marque d'étrangéité dans la traduction du quatrième traducteur, car nous estimons qu'il prend librement une décision en supprimant le nom de la ville. Par ailleurs, nous n'estimons pas qu'il existe une nécessité pour que le traducteur emploie cette technique dans cette situation. Nous sommes du même avis que Jérôme qui pense que nous pouvons tolérer les manipulations seulement quand nous en ressentons le besoin, quand on pense qu'elles vont apporter un éclairage (Ballard, 1992). Nous considérons qu'il faut se servir de cette méthode uniquement en cas d'urgence.

Tableau 4: Exemple 2 au Nom de Lieu

\begin{tabular}{lc}
\hline Livres & Extraits \\
\hline T.S. & FINOT. - Comment, et puis? Nous ferons un tas de tout ce jardinage, nous le \\
& passerons par dessus le mur, et nous irons le vendre au marché des Moulins \\
& (p.280). \\
TT1 & Fino - Sonrası var mı? İşimiz bitince duvardan atlarız; aldığımız şeyleri çarşıda \\
& satarız (p.177). \\
TT2 & Fino - Sonra ne demek? Evvelâ onları bir yere yığacağız, sonra duvarın üstünden \\
& dısarı taşıyacağız, Moulins pazarına götürüp satacağız (p.187). \\
TT3 & Finot: - Sonra da ne demek? Bu bahçe mahsullerini bir araya getirerek duvarın \\
& üstünden geçireceğiz ve gidip Moulins pazarında satacağız (p.99). \\
TT4 & - Daha ne demek? Hepsini çıkın yapıp, duvarın üstünden atar, değirmen pazarına \\
& götürüp satarız (p.153). \\
\hline
\end{tabular}

Dans ce tableau, nous rencontrons le mot "église" qui fait partie du vocabulaire de la religion chrétienne. Comme c'est une histoire qui se passe à l'étranger et que le peuple français est pratiquant de la religion catholique, il doit être très naturel d'utiliser ce mot dans les traductions aussi.

Nous remarquons que dans les première et deuxième traductions, les traducteurs traduitsent le mot tel qu'il est dans la langue source. Par contre, le troisième traducteur omet de nouveau cette partie. En effet, l'enfant lecteur n'est même pas au courant de cet évènement culturel du pays étranger. Cela peut créer des lacunes dans sa connaissance du monde.

\section{Traduction des Éléments Culturels (Transposition du Quotidien)}

Nous soutenons l'idée que Toury défend: le traducteur prend des décisions d'après les normes du pays, les effets culturels, sociaux, économiques et politiques. Dans les exemples donnés ci-dessous, nous pouvons remarquer facilement les spécificités de la langue et du registre de langue de l'époque. 
Nourriture

Tableau 5: Exemple 1 à la Nourriture

\begin{tabular}{|c|c|}
\hline Livres & Extraits \\
\hline T.S. & $\begin{array}{l}\text { Jacques. - C'est vrai! pauvre Cadichon! Et moi qui ne pensais pas à lui donner mon pain! Et, tirant } \\
\text { aussitôt de sa poche le morceau que la bonne y avait mis pour son goûter, il me le présenta. J'avais été } \\
\text { offensé de la mauvaise pensée de la bonne, et je fus bien aise de lui prouver qu'elle m'avait mal jugé } \\
(\ldots)(\text { p.88). }\end{array}$ \\
\hline TT1 & $\begin{array}{l}\text { Jak - Zavallı Kadişon! Ben ona ekmek vermeyi unuttum. Jak, cebinden dadısının koyduğu ekmeği } \\
\text { çıkararak bana uzattı. Dadının muhakkirane düşüncesinden müteessir olmuştum (p.63). }\end{array}$ \\
\hline TT2 & $\begin{array}{l}\text { Jacques - « Bu doğru! Zavallı Cadichon! Ona ekmeğimi vermeği düşünmemiştim! » Dadının ikindilik } \\
\text { olarak onun cebine koyduğu ekmek parçasının hemen çekerek bana uzattı (p.60). }\end{array}$ \\
\hline TT3 & $\begin{array}{l}\text { Jacques: -Sahi! Zavallı Cadichon! Ben de ekmeğimi ona vermeyi aklımdan bile geçirmiyordum. } \\
\text { Hizmetçinin ikindi kahvaltısı için cebine koyduğu ekmek parçasını çıkardı, bana ikram etti (p.49). }\end{array}$ \\
\hline TT4 & $\begin{array}{l}\text { Jacques: - Doğru. Zavallı Marsıvan! Cebimdeki ekmeği vermek de hiç aklıma gelmedi! Dadısının } \\
\text { kahvaltı için cebine koyduğu ekmeği çıkartıp bana uzattı (p.45). }\end{array}$ \\
\hline
\end{tabular}

Dans la première traduction, le traducteur préfère ne pas traduire le mot "goûter". Nous considérons le mot "goûter" comme un mot représentant spécifiquement la culture française. Ceci apparaît comme un détail, une nouveauté intéressante à découvrir pour l'enfant lecteur. Nous trouvons qu'en employant la stratégie de domestication (dans les trois autres traductions, le mot apparaît autrement) le traducteur fait disparaitre la culturalité.

Voici un exemple de langue des années 1940. “... muhakkirane düşüncesinden müteessir olmuştum". Ce n'est pas une expression qui est utilisée souvent de nos jours. Comme la langue change d'une époque à l'autre, et cela est surtout valable pour la langue turque, cela fait partie des indices de l'époque, un point important d'après la théorie de polysystème. N'oublions pas que la langue et les stratégies des traducteurs font partie des éléments sur lesquels on peut s'appuyer pour obtenir une image de la politique de la langue de l'époque.

Tableau 6: Exemple 2 à la Nourriture

\begin{tabular}{|c|c|}
\hline Livres & Extraits \\
\hline T.S. & $\begin{array}{l}\text { Nous arrivions au château; chacun descendit de voiture et alla défaire sa belle toilette; on m'enleva } \\
\text { aussi mes pompons, mes dahlias, et je revins brouter mon herbe pendant que les enfants mangeaient } \\
\text { leur goûter (p.193). }\end{array}$ \\
\hline TT1 & $\begin{array}{l}\text { Biraz sonra şatoya vâsıl olduk. Herkes soyundu. Ve ev elbiselerini giyindi. Benim de püsküllerimi ve } \\
\text { çiçeklerimi çıardılar. Çocuklar kahvaltı ederken ben de bahçede otladım (p.126). }\end{array}$ \\
\hline TT2 & $\begin{array}{l}\text { Şatoya geldiğimiz zaman arabadan indiler, güzel elbiselerini çıkardılar benim pompon, sorguç ve } \\
\text { yıldızlarımı da aldılar. Çocuklar ikindilik yerken bende bahçede otladım (p.125). }\end{array}$ \\
\hline TT3 & Partie Omise \\
\hline TT4 & $\begin{array}{l}\text { Şatoya geliyorduk. Her biri arabadan inip, üstlerindeki güzel elbiseleri değiştirdi. Benim de } \\
\text { pomponlarımı, yıldız çiçeklermi çkardılar. Çocuklar kahvaltı ederken, ben de gidip otlamaya } \\
\text { koyuldum (p.102). }\end{array}$ \\
\hline
\end{tabular}

Nous voyons que le premier ainsi que le deuxième traducteur adoptent le même principe, c'est-à-dire qu'ils ne traduisent pas le mot "goûter". Le mot traduit n'est pas l'équivalent du mot "goûter". Comme le troisième traducteur omet cette partie, nous ne pouvons pas argumenter. Finalement, le dernier traducteur emploie le même mot "petit déjeuner" dans la langue d'arrivée. Il utilise la même stratégie. Nous insistons sur l'importance de l'approche sourcière sur la fidélité et l'importance du choix des mots dans une traduction de la littérature de jeunesse. Dans cet exemple, nous remarquons que les traducteurs ne font pas d'effort afin de trouver l'équivalent qui existe dans la langue d'arrivée. Si nous n'arrivons pas à trouver l'équivalent du mot, nous pouvons employer d'autres stratégies pour donner le même sens. (Rappelons que Oittinen soutient l'idée de manipuler. Par contre, nous précisons qu'il faut manipuler en cas de nécessité). 
Voici de nouveau un mot reflétant l'époque de l'œuvre. "Vâsıl olmak" veut dire "ulaşmak" (Demiray, 1994: 974) en turc. Avec cet exemple, nous affirmons que les traces et les caractéristiques de l'époque apparaissent car en faisant le lien entre la traduction et son original, nous pouvons facilement décrire les spécificités de l'époque de la traduction de l'œuvre grâce à la traduction de polysystème.

Tableau 7: Exemple 3 à la Nourriture

\begin{tabular}{ll} 
Livres & \multicolumn{1}{c}{ Extraits } \\
\hline T.S. & $\begin{array}{l}\text { On s'assit par terre sous un vieux chêne; on étala le contenu des paniers. Il y } \\
\text { avait, comme à toutes les chasses, un pâté de volaille, un jambon, des œufs } \\
\text { durs, du fromage, des marmelades, des confitures, un gros baba, une énorme } \\
\text { brioche et quelques bouteilles de vieux vin (p.154). }\end{array}$ \\
& $\begin{array}{l}\text { Her av eğlencesinde olduğu gibi piliç, janbon, yumurta, peynir, reçel, birkaç } \\
\text { türlü pasta ve şarap vardı (p.104). }\end{array}$ \\
TT1 & $\begin{array}{l}\text { Bütün avlardaki gibi, kümes hayvanlarından yapılmış yemek, sucuk, lop } \\
\text { TT2 } \\
\text { bimurtalar, peynir, reçeller ezme eski şarap vardı (p.99). }\end{array}$ \\
& $\begin{array}{l}\text { Her avda olduğu gibi, tavuk, jambon, pişmiş yumurta, peynir, reçel gibi şeyler } \\
\text { getirilmişti (p.71). }\end{array}$ \\
TT3 & $\begin{array}{l}\text { Her ava çıkışta olduğu gibi tavuklu börek, yumurta, peynir, marmelat, reçel, } \\
\text { kocaman bir pasta, büyük bir çörek (p.81). }\end{array}$ \\
& TT4
\end{tabular}

Le troisième traducteur, à part le mot jambon, supprime l'étrangeté en donnant les mots équivalents en turc. Cela indique une incohérence dans son attitude. Il omet aussi plusieurs noms de nourriture dans les pages 99, 106 et 310. En conséquence, l'enfant lecteur va rencontrer beaucoup moins de mots relatifs au champ lexical de l'œuvre originale.

Le mot "gros baba" veut dire "un gâteau dans lequel on met du rhum et des raisins secs" dans la langue française. Le premier traducteur préfère employer un mot commun qui incarne deux choses en même temps. L'écrivain écrit deux choses: "le gros baba et une énorme brioche". Par ailleurs, le traducteur remplace ces deux mets avec un seul "birkaç türlü pasta" ce qui veut dire "plusieurs sortes de gâteaux". Finalement, il généralise les noms et il efface totalement l'étrangéité.

Le deuxième traducteur nous écrit l'équivalent du mot "une grosse brioche" en donnant son explication "kocaman kuru üzümlü tatlı bir çörek" qui veut dire "un grand gâteau avec des raisins secs". Nous trouvons qu'il est fidèle à l'original. Par ailleurs, il ne veut pas donner de détail. Plutôt que de dire que c'est un gâteau dans lequel on met du rhum, il dit : "C'est un gâteau sucré". Il ne traduit pas la nourriture "brioche". Il fait une adaptation d'un mot qui tient une grande importance de notre part. Honnêtement, nous imaginons que le traducteur ne connaissait pas la signification de ce mot. Sinon, quelle pourrait être la raison de ne pas l'indiquer comme le mot "gros baba".

\section{Religion}

Comme notre écrivain est une personne religieuse, dans l'œuvre "Les mémoires d'un Âne" nous retrouvons un grand nombre de notions, évènements de la religion. Nous considérons que cela fait partie des indices propres à l'écrivain. 
Tableau 8: Exemple 1 à la Religion

\begin{tabular}{ll}
\hline Livres & \multicolumn{1}{c}{ Extraits } \\
\hline T.S. & $\begin{array}{l}\text { Camille et Pierre, un peu embarrassés de leurs grandeurs, s'embrouillèrent en } \\
\text { disant le Credo; le curé fut obligé de les souffler (p.190). }\end{array}$ \\
TT1 & $\begin{array}{l}\text { Piyer ve Kamil duayı okurken şaşırıyorlard1. Papas, onlara duanın kelimelerini } \\
\text { söylemeye mecbur oldu (p.124). }\end{array}$ \\
TT2 & Partie ne pas traduite (p.123) \\
TT3 & Partie Omise \\
TT4 & $\begin{array}{l}\text { Birdenbire kendisini büyümüş sayan Pierre'le Camille, ad koyma türküsünü } \\
\text { söylerken şaşırdılar (p.100). }\end{array}$ \\
\hline
\end{tabular}

Cette partie n'existe pas du tout dans la troisième traduction comme on le voit dans d'autres chapitres aussi. En effet, nous remarquons que ce traducteur fait souvent des adaptations à la fois au niveau des paragraphes et des chapitres.

Le quatrième traducteur préfère écrire "ad koyma töreni" comme équivalent au mot "crédo". On trouve qu'il est fidèle à l'œuvre originale. Au lieu de supprimer ce mot comme les autres, il préfère le garder. En utilisant le mot "türkü”, le traducteur crée un effet folklorique, autrement dit, il turcalise le mot. On peut dire aussi qu'il est pour la domestication. Dans ce cas, on estime que le lecteur cible, l'enfant turc, va tomber dans une confusion puisque l'habitude culturelle "ad koyma töreni" fait partie d'une culture étrangère et que le mot "türkü" vient directement de la culture turque.

Tableau 9: Exemple 2 à la Religion

\begin{tabular}{|c|c|}
\hline Livres & Extraits \\
\hline T.S. & $\begin{array}{l}\text { (...) Les uns disaient que les ânes avaient été engloutis et enlevés par les } \\
\text { diables; les autres prétendaient que les religieuses enterrées dans la chapelle } \\
\text { s'en étaient emparées pour parcourir la terre; d'autres assuraient que les anges } \\
\text { qui gardaient le couvent réduisaient en cendre et en poussière tous les animaux } \\
\text { qui approchaient de trop près du cimetière où erraient les âmes des religieuses } \\
\text { (p.112). }\end{array}$ \\
\hline TT1 & $\begin{array}{l}\text { (...) Bazıları öteki eşeklerin şeytanlar tarafından kaçırılıp boğulduklarına kani } \\
\text { idiler. Bir kısmı da kilisede gömülü bulunan rahiplerin yeryüzünü dolaşmak } \\
\text { için eşekleri aldıklarını iddia ediyorlardı! Birtakımı da manastırı muhafaza } \\
\text { eden meleklerin mezarlığa fazla yaklaşanları küle ve toza tahvil ettiklerini } \\
\text { söylüyorlardı (p.77). }\end{array}$ \\
\hline TT2 & $\begin{array}{l}\text { (...) Bazıları eşeklerin, şeytanlar tarafından kaldırıldığını veya yutulduğunu } \\
\text { söylediler; bazıları da küçük kiliseye gömülü ermişlerin, dünyayı gezmek için } \\
\text { kaptıklarına ihtimal verdiler; bir kısmı da erenlerin ruhlarının dolaştığı } \\
\text { mezarlığa yaklaşan eşeklerin manastırı bekleyen meleklerce kül ve toza } \\
\text { çevrildiğini söylediler (p.73). }\end{array}$ \\
\hline TT3 & $\begin{array}{l}\text { (...) Bir kısmı eşeklerin şeytanlar tarafından yutulduğunu söylüyor, ötekiler } \\
\text { kiliseye gömülü rahibelerin, dünyayı dolaşmak için alıp götürdüklerini iddia } \\
\text { ediyorlardı. Başkaları, rahibelerin dolaştığı mezarlığa yaklaşan bütün } \\
\text { hayvanları koruyucu meleklerin küle ve toza çevirdiğini ileri sürüyorlardı } \\
\text { (p.62). }\end{array}$ \\
\hline TT4 & $\begin{array}{l}\text { (...) Kimi eşekleri şeytanların kaçırdığını söylüyor, kimi kilisede yatan } \\
\text { rahibelerin hayvanları alıp onlarla dünyayı dolaşmaya çıkacaklarını ileri } \\
\text { sürüyordu; kimine göre de koruyucu melekler mezarlığa çok yaklaşan } \\
\text { hayvanları toz haline getirmişlerdi (p.58). }\end{array}$ \\
\hline
\end{tabular}

Le premier traducteur traduit le mot tel qu'il est. Il est fidèle à l'œuvre originale. Par contre, on voit qu'il se trompe au niveau du genre du mot. À la place du mot "rahibelerin", il utilise le mot "rahiplerin". 
Les quatre traducteurs sont fidèles à l'original. Ils ne font pas d'adaptation. Nous pouvons les considérer comme "sourciers". Grâce à leur stratégie, notre lecteur "enfant" pourrait imaginer que l'histoire se déroule dans un monde chrétien à l'aide des personnages de la religion. Avec la stratégie de foreignisation, les traducteurs ont gardé la fonction éducative de l'œuvre. En effet, l'enfant pourrait s'informer sur la culture chrétienne et les habitudes des chrétiens à l'église.

Tableau 10: Exemple 3 à la Religion

\begin{tabular}{|c|c|}
\hline Livres & Extraits \\
\hline T.S. & $\begin{array}{l}\text {-Maman, dit Madeleine, est-ce que vous aimez, vous, cet usage de jeter aux } \\
\text { enfants des dragées et des centimes? LA MAMAN. - Non, chère enfant, je } \\
\text { trouve cela ignoble: les enfants deviennent semblables à des chiens qui se } \\
\text { battent pour un os. Si jamais, je suis marraine dans ce pays-ci, je ferai donner } \\
\text { des dragées, et je ferai porter aux pauvres l'argent qu'on dépense en } \\
\text { centimes, perdus en grande partie (p.192). }\end{array}$ \\
\hline TT1 & $\begin{array}{l}\text { Yolda (Madlen) annesine hitaben: - Anneciğim, bu şeker atmak âdetini nasıl } \\
\text { buluyorsunuz? Annesi - Bu âdetin aleyhindeyim. Çocuklar bir kemik için } \\
\text { dövüşen köpeklere benziyorlar. Eğer ben vaftiz annesi olursam şekeri küçük } \\
\text { külâhlara koydurup çocuklara öyle veririm. Paraları da bu suretle taksim } \\
\text { ederim (p.125). }\end{array}$ \\
\hline TT2 & $\begin{array}{l}\text { Madeleine - Anne, badem şekerlerini, ufaklık paraları çocuklara atmak adeti } \\
\text { hoşuna gidiyor mu? Anne - Hayır yavrum, çirkin buluyorum. Çocuklar, bir } \\
\text { kemik için köpeklere benziyorlar. Eğer ben isim annesi olursam badem } \\
\text { şekerlerini çocuklara, paraları da muhtaç fakirlere dağıttıracağım (p.124). }\end{array}$ \\
\hline TT3 & Partie Omise \\
\hline TT4 & $\begin{array}{l}\text { Madeleine: - Anneciğim, dedi, çocuklara böyle şeker serpmekten zevk alıyor } \\
\text { musun? Annesi: - Hayır, yavrum, diye cevap verdi. Bu davranışı çok çirkin } \\
\text { buluyorum. Çocuklar bir kemik parçası içn birbirlerini yiyen köpeklerden } \\
\text { farksız sanki ... Ben isim annesi olursam, badem şekerlerini çocuklara } \\
\text { verdireceğim, parayı da fakirlere gönderirim (p.101). }\end{array}$ \\
\hline
\end{tabular}

À part le troisième, les trois traducteurs réalisent des traductions assez fidèles à l'œuvre originale. Le mot "taksim ederim" nous renvoie directement aux années 1940. Ce mot signifie "bölüştürme" dans la nouvelle langue en turque (Demiray, 1981: 893). "Diviser, partager" sont les équivalents de ce mot. Comme c'est le cas dans plusieurs exemples, cette partie empreinte de terminologie religieuse est omise.

\section{Vêtements}

Tableau 11: Exemple 1 au Vêtement

\begin{tabular}{|c|c|}
\hline Livres & Extraits \\
\hline T.S. & $\begin{array}{l}\text { (...) À la fin du bain, l'enfant en avait assez et témoigna une vive satisfaction quand ses quatre } \\
\text { protectrices la firent sortir de la baignoire; elles la frottèrent, pour l'essuyer, jusqu'à lui faire rougir la } \\
\text { peau, et ce ne fut qu'après l'avoir séchée comme un jambon, qu'elles lui mirent une chemise, un jupon } \\
\text { et une robe de Thérèse. Tout cela allait assez bien, parce que Thérèse portait ses robes très courtes, } \\
\text { comme le font toutes les petites filles élégantes, et que la petite mendiante devait avoir ses jupons } \\
\text { tombant sur les chevilles: la taille était bien un peu longue, mais on n'y regarda pas de si près; tout le } \\
\text { monde était content (p.134). }\end{array}$ \\
\hline
\end{tabular}

(...) Artık kızcağız hamamdan çıkmak istiyordu. Dördü birden vücudunu kızartıncaya kadar oğdular. İyice kuruttuktan sonra Terezin eskilerini giydirdiler. Her şey iyi geldi; çünkü Terez zengin ve şık aile kızları gibi gayet kısa elbise giymiyordu. Küçük dilenci ise uzun entari giymeliydi! Elbisenin beli biraz boldu. Fakat kimse dikkat etmedi (p.92). kaldığını ve yıkandığını anlatmak istedi; onlar dersini çıkartıncaya kadar ovup kuruladılar. Thérèse'in bir gömleğini, bir etekliğini, bir entarisini giydirdiler. Bütün şık küçük kızlar gibi Thérèse de çok kısa 
giyindiği için elbiseler tamam geldi. Esasen küçük dilencinin etekliğinin topuklarına kadar uzun olması lâzımdi; Halbuki sırtındaki biraz uzundu. Ama kimse buna bakmıyordu (p.86).

TT3

Partie Omise

TT4 En sonunda, çocuk sıkılmaya başladı. Sudan çıkarmaya karar verdikleri zaman pek sevindi. Kurulamak için sıkı sıkı ovaladıkları için derisi kıpkırmızı olmuştu. En sonunda, Therese'in iç çamaşırlarıyla bir de entarisini giydirdiler. Hepsi de ne güzel uymuştu! Therese, zarif küçük hanımlar gibi kısa elbiseler giyiyordu. Dilenci kız ise, ayak bileklerine kadar uzanan eteklikle gezmekteydi. Boyu biraz uzun gelmişti, ama o kadar şeye önem vermediler (p.69).

Le premier traducteur utilise le mot "hamam" comme équivalent du mot "baignoire". Dans le dictionnaire de Kocabay (2001: 400) on donne "bain public" comme définition. Ce mot symbolise plutôt la culture turque. En effet, en employant un terme de culture cible, le traducteur reflète qu'il est cibliste, qu'il est pour la domestication. En lisant cette partie, préférant cette approche, le traducteur veut que l'enfant lecteur croie que l'histoire se passe en Turquie.

\section{Heure}

Tableau 12: Exemple 1 sur l'Heure

\begin{tabular}{|c|c|}
\hline Livres & Extraits \\
\hline T.S. & $\begin{array}{l}\text { Ce ne fut pas le moins amusant de l'affaire, et la vaisselle n'était pas encore } \\
\text { finie quand l'heure de l'étude sonna, et que les parents rappelèrent leurs } \\
\text { enfants pour se mettre au travail. Ils demandèrent un quart d'heure de grâce } \\
\text { pour achever de tout essuyer et ranger. On le leur accorda. Avant que le } \\
\text { quart d'heure fût écoulé, tout était rapporté à la cuisine (...) (p.323). }\end{array}$ \\
\hline TT1 & $\begin{array}{l}\text { Yemek bitince sofra toplanıldı. Çocuklar bulaşık yıkamaya başladılar. Ders } \\
\text { vakti geldi, onlar hâlâ işlerini bitirmemişlerdi. Bir çeyrek müsaade istediler; } \\
\text { lâkin bu müddet bitmeden evvel herşey yerli yerince mutfağa ve yemek } \\
\text { odasına konulmuştu. (p.201) }\end{array}$ \\
\hline TT2 & $\begin{array}{l}\text { Bu iş de az eğlendirici olmadı, okuma saati geldiği zaman bulaşık bitmişti; } \\
\text { anne ve babaları onları çalışmağa çağırdı. Çocuklar kapları kurulamak ve } \\
\text { dizmek için bir çeyrek saat izin istediler, muvafakat edildi. Çeyrek saat } \\
\text { geçmeden, her şeyi mutfağa taşıdılar (p.218). }\end{array}$ \\
\hline TT3 & $\begin{array}{l}\text { (...) Bu o kadar eğlenceli bir iş değildi. Arkasından ders saati gelip çattı. } \\
\text { Çocuklar çalışmaya gidiyorlardı, Auguste de evine dönmek için onlarla } \\
\text { vedalaştı (p.108). }\end{array}$ \\
\hline TT4 & Partie omise bütün bölüm yok \\
\hline
\end{tabular}

Nous remarquons que le premier traducteur préfère n'être pas fidèle au texte original car il manipule les phrases dans sa traduction. La phrase « les parents rappelèrent leurs enfants pour se mettre au travail » est omise. Si nous analysons la phrase: "Bir çeyrek müsaade istediler". Le terme "bir çeyrek" veut dire "un quart", et il n'est pas utilisé de nos jours. Dans le dictionnaire (Demiray, 1980: 199), on trouve l'explication "15 minutes de temps". Quant au mot "müsaade", il veut dire "permission, autorisation". Ce sont des mots qui représentent la langue spécifique de l'époque en question. Par conséquent, nous constatons que c'est l'idée directrice des recherches de la théorie du polysystème.

Comme le troisième traducteur fait plusieurs omissions de phrases, nous ne rencontrons pas la traduction de l'heure dans cette version. Cette partie est omise entièrement par le dernier traducteur. En d'autres termes, il fait preuve d'une adaptation. 
Tableau 13: Exemple 2 sur l'Heure

\begin{tabular}{ll}
\hline Livres & \multicolumn{1}{c}{ Extraits } \\
\hline T.S. & (...) Elles furent près d'un quart d'heure à arranger une compresse et la bande (...) (p. 135). \\
TT1 & (...) Ancak üç çeyrek saatte çocuğun ayağını bağlıyabildiler (...) (p.92). \\
TT2 & (...) Ancak bir çeyrek saatte sargıyı sarabildiler(...) (p.86) \\
TT3 & Partie Omise \\
TT4 & (...) Merhemi sürmek, bağ bağlamak on beş dakikadan çok sürmüştü. (...)(p. 69). \\
\hline
\end{tabular}

Le deuxième traducteur utilise le mot "bir çeyrek saatte" dans sa traduction comme équivalent au terme "un quart d'heure". Le traducteur est fidèle au texte original. À la fois, cet emploi est un indice de fidélité de l'époque de la traduction, ce qui importe pour la théorie de polysystème.

Dans la troisième traduction, le traducteur élimine cette partie en entier. Ainsi, il se permet de faire une adaptation. Ainsi, le lecteur ne va pas pouvoir lire ces termes propres à l'œuvre originale.

Le quatrième traducteur emploie le mot " 15 minutes", terme plutôt familier. Au lieu du mot "arranger la bande", il emploie "bağ bağlamak". Ce mot n'est pas son équivalent. Comme le terme "merhemi sürmek" n'existe pas du tout dans la version originale, le traducteur fait un ajout en utilisant le terme équivalent de "mettre de la pommade".

\section{Argent}

Tableau 14: Exemple 1 sur l'Argent

\begin{tabular}{ll}
\hline Livres & \multicolumn{1}{c}{ Extraits } \\
\hline T.S. & $\begin{array}{c}\text { PIERRE. - Pour quoi faire tous ces centimes? Il y en a presque autant que de dragées } \\
\text { (p.186). CAMILLE. - C'est pour jeter aux enfants de l'école (p.186). }\end{array}$ \\
TT1 & Piyer - Ne çok para!... Şekerden fazla ufak para var, bunlar neye yarar (p.122). \\
TT2 & Pierre - Şeker kadar ufaklık para var; bunlar ne olacak? (p.121) \\
TT3 & Partie Omise \\
TT4 & Pierre: - Ya bu paralar ne olacak? Badem şekeri kadar da para var (p.98) \\
\hline
\end{tabular}

Le premier traducteur n'utilise pas le mot "centime". En d'autres termes, il ne se sert pas de la stratégie de l'emprunt. Par contre, dans les autres exemples, il ne fait que le contraire, donc, on trouve qu'il n'est pas cohérent. Nous estimons que de nos jours, nous n'employons pas le terme "ufaklık para". Certainement, ce terme était employé en 1941. À la place, on écrit "ufak para". De ce fait, le deuxième traducteur utilise un lexique de la langue turque de son époque, ce qui nous réfère de nouveau à la théorie polysystémique.

Tableau 15: Exemple 2 sur l'Argent

\begin{tabular}{ll}
\hline Livres & \multicolumn{1}{c}{ Extraits } \\
\hline T.S. & $\begin{array}{l}\text { (..) C'est bon! Voilà une bonne charge qui va nous donner quelques pièces } \\
\text { de cinq francs. (p.10) }\end{array}$ \\
TT1 & (...) Bize elli kuruş hasılat verecek bir yük. (p.11) \\
TT2 & (...)Tamam işte, bize bir kaç frank getirecek iyi bir yük. (p.8) \\
TT3 & (...) Hepsi tamam. İşte bize bol bol para getirecek iyi bir yük. (p.5) \\
TT4 & (...) Tamam hiç değilse bize bize beş frank getirecek kadar mal var...(p.2)
\end{tabular}

Le premier traducteur utilise le mot "kuruş" à la place du mot "franc". Il se sert de la stratégie de "domestication". De plus, il fait une manipulation: au lieu de dire "quelques pièces de cinq", il 
préfère écrire " 50 kuruş". Nous n'arrivons pas à comprendre la raison qui le pousse à changer des mots au lieu d'employer juste l'équivalent dans la langue d'arrivée s'il existe comme c'est le cas. Dès lors, nous trouvons ce choix "inutile". En effet, le même traducteur garde le mot "franc" dans d'autres pages comme par exemple à la page 55; il préfère maintenir l'exotisme. Finalement, il est certain que ce traducteur est incohérent dans sa stratégie de traduction sur le lexique dans la catégorisation de "l'argent".

Quant au deuxième traducteur, il choisit la traduction littérale, c'est-à-dire, reste fidèle à l'ordre des mots et à la structure grammaticale. Le deuxième traducteur a utilisé le mot "frank" dans sa traduction jusqu'à la page 38 où il emploie le mot "kuruş". "Çocuklardan biri: - Bedava olmaz! dedi. Belediyenin kesesine hiç olmazsa elli kuruş koymanız gerek!”. Dans ce cas, nous considérons que cela peut causer une confusion dans l'esprit des enfants: lorsque l'enfant turc lit cette phrase, il va directement penser que l'histoire se passe dans son pays natal, la Turquie.

\section{Conclusion}

Tout au long de ce travail, plusieurs sujets ont été mis en évidence: la littérature de jeunesse, la traduction de la littérature de jeunesse, les théoriciens ciblistes, la théorie de Venuti, l'analyse comparative des quatre traductions du livre "Les mémoires d'un Àne », la classification de Göte Klingberg, Emile Benveniste et Grit et Holmes, les anthroponymes, les toponymes, les éléments culturels (la nourriture, les vêtements, la religion, les heures, les mesures) et l'adaptation.

Quand nous considérons l'aspect éducatif d'une œuvre de la littérature de jeunesse, nous nous posons la question suivante en tant que traducteur: Comment pouvons-nous manipuler l'œuvre d'une autre personne? Cela nous renvoie à la grande responsabilité du traducteur de la littérature de jeunesse. Quand il s'agit d'un jeune public, nous savons tous que le travail demande toujours plus d'attention. En étant cibliste, en employant la stratégie de domestication, comment pourrons-nous transmettre les points éducatifs, culturels de l'œuvre originale au public visé ?

Riita Oittinen, théoricienne cibliste, suggère aux traducteurs de la littérature de jeunesse de prendre totalement en compte le public visé. Dans ce cas, elle donne l'autorisation de faire des adaptations dans le texte source pour ne pas donner lieu à l'incompréhension du public cible. (Nous pensons que l'enfant est prêt à tout comprendre dès que cela lui est expliqué). Quant à nous, nous considérons que rien ne doit empêcher de se comporter sourcier même dans la traduction de la littérature de jeunesse. Il est évident que le traducteur doit s'adresser correctement aux lecteurs enfants. Par contre, nous croyons qu'il est possible de le faire en préservant les notions étrangères, tout en gardant pour objectif d'apprendre à l'enfant lecteur les nouveautés et la richesse culturelle de l'œuvre, toujours à partir de son état original. Il suffit simplement de se rappeler qu'un enfant est toujours enclin à apprendre des nouveautés.

En adoptant une attitude sourcière dans la traduction de la littérature de jeunesse, il est possible que nous forcions le traducteur à être plus créatif. Cela faisant partie d'une des missions de la littérature de jeunesse, l'enfant apprend les nouveautés. Il a la possibilité de découvrir un nouveau monde et de développer ses connaissances. Au fur et à mesure que nous selectionnons les mots corrects, le lecteur aura le même ressenti et va acquérir le même bagage linguistique que le lecteur $\mathrm{du}$ texte original. C'est la raison pour laquelle nous pensons qu'un traducteur de jeunesse devrait réagir autrement qu'en étant cibliste. En cas de nécessité, le traducteur pourra ajouter des explications, des images pour faciliter la compréhension du nouveau lexique. Ainsi, le lecteur « enfant » va pouvoir s'enrichir grâce à une traduction.

Ainsi que dans chaque œuvre de la littérature de jeunesse, "Les mémoires d'un Âne" est un livre assez riche du point de vue culturel et il nous semble qu'il importe de préserver cette richesse culturelle dans la traduction. Nous relevons des notions culturelles, à savoir des noms de plats, de nourriture spécifique à la culture française, des noms du vocabulaire spécifique à la religion et aux 
rituels religieux, à la vie quotidienne, aux coutumes non seulement des villageois et aussi de la noblesse, propre à la France de l'époque 1860.

Effectivement, dans la mesure du possible, à l'aide d'une traduction fidèle, l'enfant lecteur de la culture cible aura aussi l'occasion de pouvoir bénéficier de la richesse de l'œuvre originale. Dans le cas d'une traduction réalisée sous l'approche cibliste, sous l'objectif de la stratégie de domestication, nous considérons que l'œuvre originale, "Les mémoires d'un Âne" perdra de sa richesse littéraire et éducative pour l'enfant lecteur.

Les écrivains rédigent leurs œuvres dans leur propre langue selon leurs objectifs personnels. Nous nous posons la question suivante: est-ce qu'un livre doit être soumis à autant de manipulations durant la traduction? Personnellement, nous pensons que le traducteur devrait pouvoir transmettre les informations en préservant l'originalité de l'écrivain et de l'oeuvre. Ce qui, nous le pensons, n'empêcherait pas ces œuvres traduites d'être populaire dans une langue et culture étrangère. Par ailleurs, rappelons, en terminant ce travail, que nous travaillons dans un domaine assez particulier: "les enfants, la jeunesse". Nous considérons que "traduire un roman et écrire un roman" sont deux actes différents. Une traduction tout comme une oeuvre originale doit avoir ses propres qualités. Du point de vue de la manipulation, de l'adaptation, nous pensons qu'il faut savoir en évaluer les limites.

Pour conclure et eu égard à ces commentaires, nous comptons avoir apporté une contribution aux travaux portant sur la traduction de la littérature de jeunesse en insistant sur l'importance d'être sourcier dans ce domaine que nous qualifions de délicat, car c'est «l'enfant lecteur » qui se trouve au centre de ces échanges culturels et linguistiques.

\section{Bibliographie}

Alipui, E. (2004). Analyse polysystémique des sous-titres français du film de Woody Allen Boradway Danny Rose (1984). Mémoire présenté au département d'études françaises. Maîtrise et Arts, Institut de Traductologie.

Ballard, M. (1992). De Cicéron à Benjamin. Traducteurs, traductions, réflexions. Presses Universitaires de Lille, https://doi.org/10.7202/003509ar

Tokalak-Baltac1, F. (2017). Étude comparative des quatre traductions en turc du livre "les mémoires d'un âne " d'après différentes Théories de la traduction. (Bir eşeğin anıları » eserinin türkçeye yapılmış dört çevirisinin çeşitli çeviri kuramlarına göre incelenerek yapılan karşılaştırmalı analizi) Thèse de Doctorat, Université de Gazi. Institut des Sciences Sociales, Juin, https://doi.org/10.29228/turkishstudies.40175

Benveniste, E. (1974). Problèmes de linguistique générale, 2. Gallimard.

Boutevin, C. \& Richard-Principalli, P. (2008). Dictionnaire de la Littérature de Jeunesse. À l'usage des professeurs des écoles. Magnard-Vuibert, https://doi.org/10.7202/1008997ar

Debombourg, H. (2011). Les différents procédés de traduction dans la littérature de jeunesse. La Clé des Langues. Cultures et langues étrangères. http://cle.ens-lyon.fr/anglais/les-differentsprocedes-de-traduction-dans-la- litterature- dejeunesse121391.kjsp?RH=CDL_PLU 130000, https://doi.org/10.3726/978-3-0352-6279-7/6

De Ségur, C. (1941). Bir eşeğin hâtıratı. (Traduit par M. S. Sander). Kültür.

De Ségur, C. (1948). Bir eşeğin hatıraları. (Traduit par N. Taşer).

De Ségur, C. (1959). Bir eşeğin hattraları. (Traduit par S. Erginer).

De Ségur, C. (2006). Bir eşeğin anıları. (Traduit par G. Sayar). Türkiye İş Bankası Kültür. 
Demiray, K. (1980). Temel Türkçe sözlük. İnkılap.

Doğru, G. (2011, Mayıs). Çevirmenin görünürlük arayışı üzerine bir deneme. Evire Çevire Dergisi. Boğaziçi

Üniversitesi. https://www.academia.edu/5337619/\%C3\%87evirmenin_G\%C3\%B6r\%C3\%BCn\%C3\%B $\mathrm{Crl} \% \mathrm{C} 3 \% \mathrm{BCk}$ Aray $\% \mathrm{C} 4 \% \mathrm{~B} 1 \% \mathrm{C} 5 \% 9 \mathrm{~F} \% \mathrm{C} 4 \% \mathrm{~B} 1$ \% $\% 3 \% 9 \mathrm{Czerine}$-Bir_Deneme

Erten, A. (2011). Çocuk yazını çevirisine yaklaşımlar. Hacettepe Üniversitesi.

Escarpit, D. (2008). La literature de Jeunesse. Itinéraires d'hier à aujourd'hui. Magnard, https://doi.org/10.7202/044007ar

Ewers, H. (2009). Fundamental concepts of children's literature research: Literary and sociological approaches. New York: Routledge.

Gile, D. (2005). La traduction. La comprendre, l'apprendre. PUF, https://doi.org/10.3917/puf.gile.2005.01

Kocabay, Y. (2001). Türkçe-Fransızca sözlük.

Le Nouveau Petit Robert de la Langue Française (2008). Fransızca sözlük. Le Robert.

Oittinen, R. (2000). Translating for children. Garland.

Oseki-Dépré, I. (1999). Théories et pratiques de la traduction littéraire. Armand ColinPederzoli, R. (2012). La traduction de la littérature de jeunesse et le dilemme du destinataire. Peter Lang, https://doi.org/10.3726/978-3-0352-6279-7/9

Rakova, Z. (2014). Les théories de la traduction. Masarykova univerzita. Brno. https:/digilib.phil.muni.cz/data/handle/11222.digilib/130676/monography.pdf

Torres, M. C. (2012). Parlons du traducteur: rôle et profil. http://traduire.revues.org/479, https://doi.org/10.4000/traduire.479

Witteveen, J.G. (2012). Le Clézio. Le chercheur d'or. Mémoire de Maitrise, Université d'Utrecht, Faculté des Sciences Humaines. 